\title{
Phenotypic Detection of Genitourinary Candidiasis among Sexually Transmitted Disease Clinic Attendees in Ladoke Akintola University Teaching Hospital, Osogbo, Nigeria
}

\author{
Oluranti J. Obisesan, ${ }^{1}$ Olugbenga A. Olowe, ${ }^{2}$ and Samuel S. Taiwo ${ }^{2}$ \\ ${ }^{1}$ Department of Medical Microbiology and Parasitology, Ladoke Akintola University of Technology Teaching Hospital, \\ PMB 5000, Osogbo, Nigeria \\ ${ }^{2}$ Department of Medical Microbiology and Parasitology, College of Health Sciences, Ladoke Akintola University of Technology, \\ PMB 4400, Osogbo, Nigeria
}

Correspondence should be addressed to Olugbenga A. Olowe; oaolowe@lautech.edu.ng

Received 10 February 2015; Revised 17 April 2015; Accepted 20 April 2015

Academic Editor: Ike S. Okosun

Copyright (C) 2015 Oluranti J. Obisesan et al. This is an open access article distributed under the Creative Commons Attribution License, which permits unrestricted use, distribution, and reproduction in any medium, provided the original work is properly cited.

\begin{abstract}
The management of genitourinary candidiasis (GC) is fraught with challenges, especially, in an era of increasing antifungal resistance. This descriptive cross-sectional study conducted between May 2013 and January 2014 determined the prevalence and characteristics of GC and the species of Candida among 369 attendees of a Sexually Transmitted Disease (STD) clinic of Ladoke Akintola University Teaching Hospital, Osogbo, Nigeria. Appropriate urogenital specimen collected from each attendee was examined by microscopy and culture for Candida, with preliminary species identification by CHROMAgar Candida and confirmation by Analytical Profile Index (API) 20C AUX. The age range of attendees was 1-80 years, mean age was $36.32 \pm 11.34$ years, and male to female ratio was 1 to 3 . The prevalence of genitourinary candidiasis was $47.4 \%$, with $4.9 \%$ in males and $42.5 \%$ in females $(p<0.0001)$. The age groups 31-45 and 16-30 have the highest prevalence of $23.3 \%$ and $16.8 \%$, respectively. The species of Candida recovered include Candida glabrata 46.9\%, Candida albicans 33.7\%, Candida dubliniensis 9.7\%, Candida tropicalis 5.7\%, Candida krusei $1.7 \%$, Candida lusitaniae $1.7 \%$, and Candida utilis $0.6 \%$. This study reported non-C. albicans Candida, especially $C$. glabrata, as the most frequently isolated species in GC, contrary to previous studies in this environment and elsewhere.
\end{abstract}

\section{Introduction}

Genitourinary candidiasis is a fungal infection of the genital and urinary tracts caused by Candida, presenting as vulvovaginal candidiasis in women, balanitis and balanoprostitis in men, and candiduria in both sexes $[1,2]$. Candida species are normal microbiota of the respiratory tract, gastrointestinal tract, mouth, and vagina, and cause opportunistic infections when altered host conditions enable the fungus to proliferate. Although genitourinary candidiasis can occur in both immunocompetent and immunocompromised persons, it is a cause of morbidity and mortality in immunocompromised patients in particular [3]. Risk factors associated with genitourinary candidiasis include sexual contacts, poor genital hygiene, urinary instrumentation, urinary obstruction, and incomplete bladder emptying [4-6]. Among Candida species implicated, epidemiological studies have shown that C. albicans is the most commonly isolated species [6-9]. The newly emerging non-Candida albicans Candida, including Candida glabrata, Candida krusei, Candida tropicalis, Candida lusitaniae, and Candida parapsilosis, are however now implicated as causative agents [10-14] of genitourinary candidiasis.

The incidence of genitourinary candidiasis varies in relation to gender, host susceptibility, and hospital settings. The incidence is higher in women and among hospitalized patients [7-9], particularly in intensive care [7] and burn units [15]. The incidence is also high in pregnant women and in HIV-infected patients [16, 17], in patients with uncontrolled diabetes mellitus [18-21], and in individuals with multiple sexual partners $[5,16,22]$. 
Genitourinary candidiasis can be symptomatic or asymptomatic $[23,24]$. Vulva pruritus and vaginal discharge are the most frequent symptoms of vulvovaginal candidiasis [25]. The discharge, which is usually adherent but not offensive, is typically cottage cheese-like in appearance but may range from watery to homogenously thick discharge. Other symptoms include vaginal soreness from scratching, vulva burning, dyspareunia, and dysuria. There may be erythema and swelling of the labia and vulva on examination but the cervix usually appears normal [25, 26]. Asymptomatic candiduria often occurs in hospitalized patients with indwelling catheters, and these patients usually show none of the signs or symptoms associated with urinary tract infection [3]. Although candiduria frequently occurs, especially in catheterized patients, symptomatic candida cystitis is rare, as bladder is relatively resistant to Candida invasion. When it occurs, however, it may present with signs and symptoms of bladder irritation including dysuria, hematuria, frequency, urgency, and suprapubic tenderness [27]. Conversely, symptomatic candida cystitis is rare in noncatheterized patients; however prostatic abscess caused by Candida species is not uncommon in male patients with diabetes mellitus $[15,28]$.

In our clinic, the diagnosis of genitourinary candidiasis is often made on clinical ground and by conventional laboratory detection of Candida with wet film/Gram smear microscopy, and culture on Sabouraud Dextrose Agar followed by identification based on colonial morphology, germ tube test, and formation of chlamydospores on cornmeal agar [27]. However other non-C. albicans species such as C. glabrata and C. dubliniensis are known to exhibit morphologic and biochemical characteristics indistinguishable from C. albicans. This conventional method therefore may not differentiate the Candida species, and this gives tendency to overreporting of $C$. albicans as the most frequent cause of genitourinary candidiasis.

In view of the changing epidemiology of fungi infections occasioned by worldwide increased incidence of immunocompromising diseases coupled with increased use of immunosuppressive therapies and resistance to traditional antifungi agents [29], it is imperative to employ methods that will identify species of Candida that may exhibit different susceptibility to currently available antifungi agents. These methods include the use of selective chromogenic media such as CHROMAgar which allow rapid identification of different Candida species based on colour changes produced from utilization of chromogenic substrates, facilitating detection of even mixed infections with more than one Candida species [29]. Commercial identification kits such as the Analytical Profile Index (API) that measures metabolic activities of Candida, ELISA antigen detection, and molecular methods such as the Polymerase Chain Reaction (PCR) tests can reliably differentiate $C$. albicans from non-C. albicans Candida species $[11,13]$. However, only few laboratories in our environment have financial capability to employ these methods for routine identification of Candida isolates.

In this study, we employed conventional culture for initial isolation of Candida and identify the species of Candida using CHROMAgar Candida and then confirmed the species by the API 20C AUX. The objectives are to determine the prevalence and characteristics of genitourinary candidiasis as well as the species of Candida among attendees of our Sexually Transmitted Disease (STD) clinic. This information is necessary for proper management of genitourinary candidiasis in view of recent reports in our hospital of apparent failed treatment among women treated for vaginal candidiasis with azole antifungi agents.

\section{Materials and Methods}

2.1. Study Design and Setting. A descriptive cross-sectional study was conducted during the period May 2013 and January 2014 at Ladoke Akintola University Teaching Hospital, Osogbo, Nigeria. The research project proposal was approved by the Ethical Committee of the institution. Informed consent was obtained from each attendee prior to the start of the study.

2.2. Subjects. All consenting attendees (all age groups and gender) of the Sexually Transmitted Disease (STD) clinic for purposes of evaluation on account of STDs or genitourinary complaints were recruited into the study. No attendee was excluded except those who did not give informed consent. Sociodemographic and clinical data of each attendee were collected into an interview protocol form. Our STD clinic has recently been renamed Genitourinary Medicine (GUM) clinic to accommodate all cases of genitourinary complaints aside STDs.

2.3. Specimen Collection. Appropriate specimen types were collected in relation to gender, age, and complaints. Vulvovaginal and urethra swabs were collected by qualified medical personnel using cotton tipped swab. High vaginal and endocervical swabs were collected after speculum examination from posterior fornix and endocervix, respectively. All swabs were immediately transported to the Medical Microbiology Laboratory of the hospital for processing. For urine specimen, each adult attendee was instructed (verbal and written) to collect $10 \mathrm{~mL}$ of a clean-catch midstream urine specimen into a sterile screw-capped wide-mouth container and immediately deliver it to the laboratory. For children, parents or guardians were instructed appropriately how to collect urine specimen from the child into sterile bottle.

2.4. Wet Film Microscopy. A swab stick containing specimen was emulsified with small volume of normal saline $(0.9 \%$ $\mathrm{NaCl}$ ) on a clean grease-free glass slide and covered with a cover slip. The preparation was examined under the microscope using 10x and 40x objective lenses for oval budding yeast presumed to be Candida [27].

A deposit of centrifuged urine was placed on a glass slide, covered with a cover slip, and examined microscopically at 400x magnifications for presence of yeast cells and other cells such as trichomonads and clue cells.

2.5. Gram Stain Microscopy. Smears of swabs were made from the saline mixture and urine deposit on a clean glass slide. Each smear was air-dried and heat-fixed by passing over a Bunsen burner flame three times to fix the slide. The slide 
TABLE 1: Sociodemographic characteristics of Sexually Transmitted Disease (STD) clinic attendees in LAUTECH Teaching Hospital, Osogbo, Nigeria.

\begin{tabular}{|c|c|c|c|c|}
\hline \multirow{2}{*}{$\begin{array}{l}\text { Characteristics } \\
\text { (1) Age group (years) }\end{array}$} & \multicolumn{3}{|c|}{ Number of attendees } & \multirow[t]{2}{*}{$\%$} \\
\hline & Male & Female & Total & \\
\hline $1-15$ & 2 & 8 & 10 & 2.7 \\
\hline $16-30$ & 20 & 84 & 104 & 28.2 \\
\hline $31-45$ & 51 & 148 & 199 & 53.9 \\
\hline $45-60$ & 8 & 35 & 43 & 11.6 \\
\hline$>60$ & 10 & 3 & 13 & 3.5 \\
\hline Total & 91 & 278 & 369 & 100 \\
\hline \multicolumn{5}{|l|}{ (2) Marital status } \\
\hline Married & & 142 & & 38.5 \\
\hline Married but separated & & 58 & & 15.7 \\
\hline Married but divorced/widowed & & 75 & & 20.3 \\
\hline Single & & 94 & & 25.5 \\
\hline \multicolumn{5}{|l|}{ (3) Educational status } \\
\hline Tertiary & & 152 & & 41.2 \\
\hline Primary/secondary & & 125 & & 33.9 \\
\hline Artisan/skilled workers/uneducated & & 92 & & 24.9 \\
\hline \multicolumn{5}{|l|}{ (4) Occupational status } \\
\hline Full-time employment & & 162 & & 43.9 \\
\hline Part-time employment & & 114 & & 30.9 \\
\hline Not employed & & 93 & & 25.2 \\
\hline \multicolumn{5}{|l|}{ (5) Categories of attendees } \\
\hline Pregnant women & & 86 & & 23.3 \\
\hline HIV-infected & & 94 & & 25.5 \\
\hline $\mathrm{DM}$ & & 70 & & 18.9 \\
\hline UTI & & 33 & & 8.9 \\
\hline Apparently healthy & & 86 & & 23.3 \\
\hline
\end{tabular}

was Gram-stained using recommended procedure and then examined for oval yeast cells [27, 30].

2.6. Qualitative Culture and Identification of Candida Species. Swabs and urine specimens were inoculated onto Sabouraud Dextrose Agar (Oxoid, UK) supplemented with chloramphenicol and incubated at $37^{\circ} \mathrm{C}$ for 48 hours for qualitative culture analysis. Colonies from SDA were first Gramstained and those that showed Gram positive yeast were then subcultured on CHROMAgar Candida (Paris, France) selective and differential medium. With the inclusion of chromogenic substrates in the medium, the colonies of $C$. albicans, C. tropicalis, C. glabrata, and C. krusei produce different colors, thus allowing the detection of these Candida species. Colonies of C. albicans appeared as light to medium green, $C$. tropicalis colonies as dark blue to metallic blue, $C$. dubliniensis as dark green, C. krusei colonies as pale pink with white edge rough, $C$. glabrata as pink to violet surface with pale edges, C. lusitaniae as pink to lavender, and C. utilis as glossy pink. All Candida species identified on CHROMAgar were further subjected to sugar assimilation and fermentation tests using API Candida (API BioMérieux, France) to confirm each species. Fungi susceptibility testing could not be done due to lack of facility in our laboratory at the time of this study.

2.7. Data Entry and Statistical Analysis. Sociodemographic, clinical, and microbiological data were entered into SPSS (Statistical Package for the Social Sciences) version 16.0 on a Window 7 laptop computer for analysis. The Chi-square or Fisher exact test was used to estimate significant differences between categorical variables. Values were considered to be statistically significant when the $p$ value obtained was less than 0.05 .

\section{Results}

3.1. Study Population. A total number of 369 attendees (mean age: $36.32 \pm 11.34$ years, age range: 1 to 80 years) were studied of which $91(24.7 \%)$ were males and $278(75.3 \%)$ were females, giving a $\mathrm{M}: \mathrm{F}$ ratio of $1: 3$. The age group distribution and sociodemographic characteristics of the study population are shown in Table 1 . The age groups $31-45$ and 16-30 constitute the largest group patronizing the STD clinic with $53.9 \%$ (199 of 369) and $28.2 \%$ (104 of 369), respectively. Of the 369 attendees, 86 (23.3\%) were pregnant women, 
TABLE 2: Distribution of genitourinary candidiasis by age group and gender among STD clinic attendees of LAUTECH Teaching Hospital, Osogbo, Nigeria.

\begin{tabular}{|c|c|c|c|c|c|c|c|}
\hline \multirow{2}{*}{ Age group (years) } & \multicolumn{2}{|c|}{ Gender } & \multirow{2}{*}{ Total } & \multirow{2}{*}{ Prevalence rate (\%) } & \multirow{2}{*}{$95 \%$ CI } & \multirow{2}{*}{ OR } & \multirow{2}{*}{$p$ value } \\
\hline & Male & Female & & & & & \\
\hline $1-15$ & 1 & 6 & 7 & 1.9 & & & \\
\hline $16-30$ & 5 & 57 & 62 & 16.8 & & & \\
\hline $31-45$ & 6 & 80 & 86 & 23.3 & & & $p=0.0034$ \\
\hline $46-60$ & 1 & 12 & 13 & 3.5 & & & \\
\hline$>60$ & 5 & 2 & 7 & 1.9 & & & \\
\hline Total & 18 & 157 & 175 & 47.4 & $0.1077-0.3353$ & 0.1900 & $p<0.0001$ \\
\hline
\end{tabular}

TABLE 3: Symptomatology of genitourinary candidiasis among STD clinic attendees in LAUTECH Teaching Hospital, Osogbo, Nigeria.

\begin{tabular}{lcccc}
\hline Categories & $\begin{array}{c}\text { Number of } \\
\text { attendees }\end{array}$ & $\begin{array}{c}\text { Number of those } \\
\text { positive Candida }\end{array}$ & $\begin{array}{c}\text { Number of symptomatic } \\
\text { attendees }\end{array}$ & $\begin{array}{c}\text { Number of asymptomatic } \\
\text { attendees }\end{array}$ \\
\hline Pregnancy & 86 & 51 & 39 & 12 \\
HIV-infected & 94 & 40 & 1 & 39 \\
DM & 70 & 34 & 7 & 9 \\
UTI & 33 & 10 & 0 & 3 \\
Apparently healthy & 86 & 40 & 72 & 103 \\
\hline Total & 369 & 175 & & 40 \\
\hline
\end{tabular}

94 (25.5\%) had underlying HIV infection, 70 (18.9\%) had diabetes mellitus (DM), 33 (8.9\%) had underlying urinary tract infection (UTI), and $86(23.3 \%)$ were visiting the clinic for fear of having contacted STDs (venereophobia) but had no underlying medical condition or illness.

\subsection{Prevalence and Characteristics of Genitourinary Candidi-} asis. Table 2 shows the prevalence of genitourinary candidiasis in the study population. Of the 369 attendees, 175 were positive for Candida, giving a prevalence of genitourinary candidiasis of $47.4 \%$ (175 of 369) in the study, with $4.9 \%$ (18 of 369) in males and $42.5 \%$ (157 of 369) in females (OR 1.9, $p<0.0001$ ). The age groups $31-45$ and 16-30 which constitute the largest groups patronizing the clinic also have the highest prevalence of genitourinary candidiasis with $23.3 \%$ and $16.8 \%$, respectively $(p=0.0034)$. Symptoms of genitourinary candidiasis were seen in only 72 (41.1\%) of the 175 attendees positive for Candida which include 39 pregnant women, $1 \mathrm{HIV}$-infected individual, 25 DM patients, 7 UTI patients, and none of the apparently healthy attendees. One hundred and three (58.9\%) of those positive for Candida were asymptomatic and include 12 pregnant women, $39 \mathrm{HIV}$ infected individuals, 9 DM patients, 3 UTI patients, and 40 apparently healthy individuals (Table 3 and Figure 1).

3.3. Frequency of Isolation of Candida Species. Table 4 shows the species of Candida recovered in the study and their frequency of isolation. They include Candida glabrata 82 (46.9\%), Candida albicans 59 (33.7\%), Candida dubliniensis 17 (9.7\%), Candida tropicalis 10 (5.7\%), Candida krusei 3 (1.7\%), Candida lusitaniae 3 (1.7\%), and Candida utilis 1 (0.6\%). Both C. albicans and non-C. albicans Candida species were recovered across all categories of attendees without any particular pattern or preference $(p=0.6455>0.05$, Table 5).

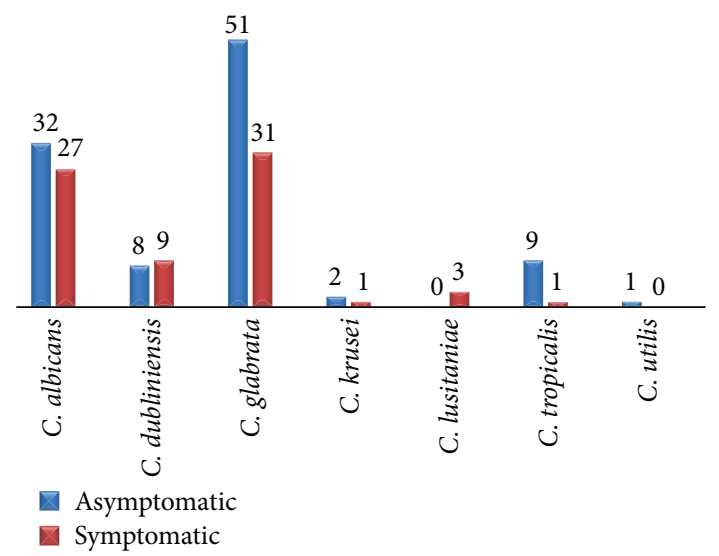

FIgURE 1: Distribution of Candida species in symptomatic and asymptomatic attendees.

TABLE 4: Frequency of occurrence of Candida species among STD clinic attendees of LAUTECH Teaching Hospital, Osogbo, Nigeria.

\begin{tabular}{lcc}
\hline Candida species & Frequency & Percentage (\%) \\
\hline Candida glabrata & 82 & 46.9 \\
Candida albicans & 59 & 33.7 \\
Candida dubliniensis & 17 & 9.7 \\
Candida tropicalis & 10 & 5.7 \\
Candida krusei & 3 & 1.7 \\
Candida lusitaniae & 3 & 1.7 \\
Candida utilis & 1 & 0.6 \\
\hline Total & 175 & 100 \\
\hline
\end{tabular}

The recovery rate of Candida species from clinical specimens was highest with urine samples followed by vulvovaginal swabs and least from urethral swab (Table 6). 
TABLE 5: Distribution of Candida albicans and non-C. albicans Candida species among STD clinic attendees of LAUTECH Teaching Hospital, Osogbo, Nigeria.

\begin{tabular}{|c|c|c|c|c|c|}
\hline Categories of attendees & C. albicans (\%) & Non-C. albicans Candida (\%) & Total (\%) & $X^{2}$ & $p$ value \\
\hline Pregnancy & 17 & 34 & 51 & \multirow{5}{*}{2.495} & \multirow{5}{*}{$p=0.6455$} \\
\hline HIV-infected & 14 & 26 & 40 & & \\
\hline $\mathrm{DM}$ & 8 & 26 & 34 & & \\
\hline UTI & 4 & 6 & 10 & & \\
\hline Apparently healthy & 16 & 24 & 40 & & \\
\hline Total & $59(33.7)$ & $116(66.3)$ & $175(100)$ & & \\
\hline
\end{tabular}

TABLE 6: Recovery rate of Candida species from clinical specimens obtained from STD clinic attendees of LAUTECH Teaching Hospital, Osogbo, Nigeria.

\begin{tabular}{|c|c|c|c|c|c|}
\hline Clinical specimens & Number of samples & Number of those positive for Candida & $\%$ Recovery rate & $X^{2}$ & $p$ value \\
\hline Vulvovaginal swab & 266 & 124 & 46.6 & & \\
\hline Urine & 78 & 50 & 64.1 & 27.678 & $p<0.0001$ \\
\hline Urethral swab & 25 & 1 & 4.0 & & \\
\hline Total & 369 & 175 & 47.4 & & \\
\hline
\end{tabular}

\section{Discussion}

The incidence of genitourinary candidiasis has dramatically increased in recent years as a result of increase in the incidence of immunodeficiency diseases such as HIV/AIDS, malignancies, metabolic dysfunctions, and the increasing use of immunosuppressive therapies for organ transplantation as well as the use of broad spectrum antibiotic therapy [29]. This study determined the prevalence and characteristics of genitourinary candidiasis as well as the species of Candida among attendees of Sexually Transmitted Disease (STD) clinic of Ladoke Akintola University Teaching Hospital, Osogbo, Nigeria. The prevalence of $47.4 \%$ reported in our study agrees with rates reported in previous studies of Enweani et al. [31], Sehgal [32], and Okungbowa et al. [33] conducted on similar populations and who recorded $40.7 \%$, $54 \%$, and $57.3 \%$ rates, respectively. However, the rate in our study was far less than the rate reported in a more recent study by Farooqi et al. [34]. The differences in rates reported may be attributed to the geographical variability, period of specimen collection, methods of identification, and differences in study population.

Candida glabrata, a non-C. albicans Candida, was the most prevalent causative agent of genitourinary candidiasis in this study with $46.9 \%$. This is in conformity with Okungbowa et al. [33] who reported C. glabrata as the most prevalent Candida species in their study with $33.7 \%$ and Srujana et al. [35] with 50.4\%. Other studies that have reported nonC. albicans as predominant isolates such as Malini [36] with $54.1 \%$ and Ragini et al. [37] with $69.7 \%$ also agree with the $66.3 \%$ of non-C. albicans Candida isolates reported in our current study. However some recent studies $[6,38]$ still reported $C$. albicans as the predominant Candida species isolated in genitourinary candidiasis. The significance of our finding is in the light of postulates by some investigators that C. glabrata may have emerged as breakthrough vaginal infections in women receiving long-term maintenance lowdose fluconazole prophylaxis [24]. There is a high possibility in our environment that C. glabrata and other non-C. albicans may have been responsible for recurrent vaginal candidiasis and apparent failed treatment seen in many women treated with ketoconazole and fluconazole, two most important antifungi azoles routinely used in our hospital for treating candidiasis (personal communication). We also noted higher Candida recovery from urine specimen, especially for the non-C. albicans Candida, than from vulvovaginal or urethral swabs. Examination of urine may therefore serve as credible alternative, especially when vulvovaginal swab cultures are persistently negative, but the patients continue to experience symptoms of genitourinary candidiasis.

In this study, genitourinary candidiasis occurred most frequently in the age groups 31-45 and 16-30 years which together had prevalence rate of $40.1 \%$. This age bracket, which encompasses teenagers, adolescents, and young adults, represents the most sexually active population group, with predisposition to increased sexual activities, an important risk factor for genitourinary candidiasis $[5,16,22,39]$. This observation has been similarly reported by other researchers in Nigeria [33, 38, 40] and elsewhere [32]. It is however interesting to note that we isolated Candida from urine of 7 out of the 10 children (age group 1-15 years) enrolled in the study, 6 of whom were prepubertal girls and 1 was a male child. All 7 had symptoms of urinary tract infection and non-C. albicans were recovered from 5 of them. Candida is a normal flora of the vagina of prepubertal girls and usually kept at bay by the lactobacillus flora. However, a distortion in the normal lactobacilli balance, especially following broad spectrum antibiotic therapy, sexual activity, or use of contraceptives, may enable Candida, especially C. glabrata, to proliferate to produce symptomatic genitourinary disease [41-43]. We could not ascertain the factors that predisposed these children to candidiasis. However, three of them had 
received antibiotic therapy two weeks before the study. The preponderance of females attending our STD clinic and the high prevalence of genitourinary candidiasis in them have previously been established in our institution [44].

Genitourinary candidiasis can be symptomatic or asymptomatic $[23,24]$. In our study, only $41.1 \%$ of the clinic attendees were symptomatic of candidiasis, most of whom were pregnant women and diabetic patients, in agreement with what has been previously reported $[19,45]$. The apparently healthy attendees (who were probably venereophobic) and the HIV-infected patients were largely asymptomatic, although Candida was isolated from their urine or vulvovaginal swabs. One limitation in our study was that we did not perform quantitative Candida culture to determine whether density of organisms correlated with symptomatology; hence we are unable to provide explanation why these groups of attendees were asymptomatic. However, it is generally established that high inoculum of fungi organisms correlate with fungi pathogenicity and symptomatology [3, 46]. Asymptomatic genitourinary candidiasis usually does not require specific treatment as correcting any risk factor can result in remission of disease. However, immunocompromised patients, who are asymptomatic carrier of Candida, are at risk of developing invasive fungal infections; hence all HIV-infected and diabetes mellitus patients in our study and all symptomatic patients were offered appropriate antifungi therapy.

\section{Conclusion}

The findings in this study showed that C. albicans, usually reported to be the most frequently isolated species in genitourinary candidiasis, was not the main species recovered. The non-C. albicans Candida, especially C. glabrata, were most frequently isolated. Phenotypic methods of identification used in this study proved adequate for the primary isolation, presumptive identification, and speciation of Candida.

\section{Conflict of Interests}

The authors declare that there is no conflict of interests regarding the publication of this paper.

\section{References}

[1] S. Edwards, "Balanitis and balanoposthitis: a review," Genitourinary Medicine, vol. 72, no. 3, pp. 155-159, 1996.

[2] C. Lisboa, A. Ferreira, C. Resende, and A. G. Rodrigues, "Infectious balanoposthitis: management, clinical and laboratory features," International Journal of Dermatology, vol. 48, no. 2, pp. 121-124, 2009.

[3] J. M. Achkar and B. C. Fries, "Candida infections of the genitourinary tract," Clinical Microbiology Reviews, vol. 23, no. 2, pp. 253-273, 2010.

[4] L. O. Eckert, S. E. Hawes, C. E. Stevens, L. A. Koutsky, D. A. Eschenbach, and K. K. Holmes, "Vulvovaginal candidiasis: clinical manifestations, risk factors, management algorithm," Obstetrics \& Gynecology, vol. 92, no. 5, pp. 757-765, 1998.
[5] M. G. Marin, R. King, S. Sfameni, and G. J. Dennerstein, "Adverse behavioral and sexual factors in chronic vulvar disease," The American Journal of Obstetrics and Gynecology, vol. 183, no. 1, pp. 34-38, 2000.

[6] E. E. Akortha, V. O. Nwaugo, and N. O. Chikwe, "Antifungal resistance among Candida species from patients with genitourinary tract infection isolated in Benin City, Edo state, Nigeria," African Journal of Microbiology Research, vol. 3, no. 11, pp. 694699, 2009.

[7] D. R. Schaberg, D. H. Culver, and R. P. Gaynes, "Major trends in the microbial etiology of nosocomial infection," The American Journal of Medicine, vol. 91, no. 3, pp. 72S-75S, 1991.

[8] C. A. Kauffman, J. A. Vazquez, J. D. Sobel et al., "Prospective multicenter surveillance study of funguria in hospitalized patients. The National Institute for Allergy and Infectious Diseases (NIAID) Mycoses Study Group," Clinical Infectious Diseases, vol. 30, no. 1, pp. 14-18, 2000.

[9] F. \&apos; Alvarez-Lerma, J. Nolla-Salas, C. Le\&apos; on et al., "Candiduria in critically ill patients admitted to intensive care medical units," Intensive Care Medicine, vol. 29, no. 7, pp. 10691076, 2003.

[10] K. C. Hazen, "New and emerging yeast pathogens," Clinical Microbiology Reviews, vol. 8, no. 4, pp. 462-478, 1995.

[11] J. Xu, C. M. Boyd, E. Livingston, W. Meyer, J. F. Madden, and T. G. Mitchell, "Species and genotypic diversities and similarities of pathogenic yeasts colonizing women," Journal of Clinical Microbiology, vol. 37, no. 12, pp. 3835-3843, 1999.

[12] K. H. Abu-Elteen, "Increased incidence of vulvovaginal candidiasis caused by Candida glabrata in Jordan," Japanese Journal of Infectious Diseases, vol. 54, no. 3, pp. 103-107, 2001.

[13] P. P. Chong, Y. L. Lee, B. C. Tan, and K. P. Ng, "Genetic relatedness of Candida strains isolated from women with vaginal candidiasis in Malaysia," Journal of Medical Microbiology, vol. 52, no. 8, pp. 657-666, 2003.

[14] L. A. Namkinga, M. I. N. Matee, A. K. Kivaisi, and C. Moshiro, "Prevalence and risk factors for vaginal candidiasis among women seeking primary care for genital infections in Dar Es Salaam, Tanzania," East African Medical Journal, vol. 82, no. 3, pp. 138-143, 2005.

[15] M.-E. Bougnoux, G. Kac, P. Aegerter et al., "Candidemia and candiduria in critically ill patients admitted to intensive care units in France: incidence, molecular diversity, management and outcome," Intensive Care Medicine, vol. 34, no. 2, pp. 292$299,2008$.

[16] A. Spinillo, G. Pizzoli, L. Colonna, S. Nicola, F. de Seta, and S. Quaschino, "Epidemiology characteristics of women with idiopathic recurrent vulvo vaginal candidiasis (VVC)," Obstetrics \& Gynecology, vol. 81, pp. 721-727, 1993.

[17] P. L. Fidel Jr., J. A. Vazquez, and J. D. Sobel, "Candida glabrata: review of epidemiology, pathogenesis, and clinical disease with comparison to C. albicans," Clinical Microbiology Reviews, vol. 12, no. 1, pp. 80-96, 1999.

[18] J. D. Sobel, "Pathogenesis and treatment of recurrent vulvovaginal candidiasis," Clinical Infectious Diseases, vol. 14, no. 1, pp. S148-S153, 1992.

[19] R. Goswami, V. Dadhwal, S. Tejaswi et al., "Species-specific prevalence of vaginal candidiasis among patients with diabetes mellitus and its relation to their glycaemic status," Journal of Infection, vol. 41, no. 2, pp. 162-166, 2000.

[20] E. M. de Leon, S. J. Jacober, J. D. Sobel, and B. Foxman, "Prevalence and risk factors for vaginal Candida colonization 
in women with type 1 and type 2 diabetes," BMC Infectious Diseases, vol. 2, article 1, 2002.

[21] R. Faraji, M. A. Rahimi, F. Rezanmadani, and M. Hashemi, "Prevalence of vaginal candidiasis infection in diabetic women," African Journal of Microbiology Research, vol. 6, no. 11, pp. 27732778, 2012.

[22] B. Foxman, "The epidemiology of vulvovaginal candidiasis: risk factors," American Journal of Public Health, vol. 80, no. 3, pp. 329-331, 1990.

[23] M. J. Richards, J. R. Edwards, D. H. Culver, and R. P. Gaynes, "Nosocomial infections in combined medical-surgical intensive care units in the United States," Infection Control and Hospital Epidemiology, vol. 21, no. 8, pp. 510-515, 2000.

[24] N. Paul, E. Mathai, O. C. Abraham, J. S. Michael, and D. Mathai, "Factors associated with candiduria and related mortality," Journal of Infection, vol. 55, no. 5, pp. 450-455, 2007.

[25] S. Natalie, "Recurrent vulvovaginal candidiasis," South African Pharmaceutical Journal, vol. 79, no. 6, pp. 14-17, 2012.

[26] T. E. Brown, L. D. Dresser, and T. W. F. Chin, "Superficial fungal infections," in Pharmacotherapy: A Pathophysiologic Approach, J. T. DiPiro, R. L. Talbert, and G. C. Yee, Eds., The McGraw-Hill Companies, New York, NY, USA, 8th edition, 2011.

[27] M. Cheesbrough, District Laboratory Practice in Tropical Countries-Part 2, Cambridge University Press, Cambridge, UK, 2000.

[28] H. W. Boucher, A. H. Groll, C. C. Chiou, and T. J. Walsh, "Newer systemic antifungal agents: pharmacokinetics, safety and efficacy," Drugs, vol. 64, no. 18, pp. 1997-2020, 2004.

[29] M. A. Pfaller and D. J. Diekema, "Rare and emerging opportunistic fungal pathogens: concern for resistance beyond Candida albicans and Aspergillus fumigatus," Journal of Clinical Microbiology, vol. 42, no. 10, pp. 4419-4431, 2004.

[30] E. van Dyck, A. Z. Mehem, and P. Piot, Laboratory Diagnosis of Sexually Transmitted Diseases, World Health Organization, Geneva, Switzerland, 1999.

[31] I. B. Enweani, C. I. C. Ogbonna, and W. Kozak, "The incidence of candidiasis amongst the asymptomatic female students of the University of Jos, Nigeria," Mycopathologia, vol. 99, no. 3, pp. 135-141, 1987.

[32] S. C. Sehgal, "Epidemiology of male urethritis in Nigeria," Journal of Tropical Medicine and Hygiene, vol. 93, no. 2, pp. 151152, 1990.

[33] F. I. Okungbowa, O. S. Isikhuemhen, and A. P. O. Dede, “The distribution frequency of Candida species in the genitourinary tract among symptomatic individuals in Nigerian cities," Revista Iberoamericana de Micologia, vol. 20, no. 2, pp. 60-63, 2003.

[34] J. Farooqi, K. Jabeen, N. Saeed, A. Zafar, M. E. Brandt, and R. Hasan, "Species identification of invasive yeasts including Candida in Pakistan: limitations of phenotypic methods," Journal of the Pakistan Medical Association, vol. 62, no. 10, pp. 995-998, 2012.

[35] M. Srujana, I. Xess, F. Hassan, A. Kapil, S. Mittal, and J. E. Tolosa, "Prevalence and susceptibility to fluconazole of Candida species causing vulvovaginitis," The Indian Journal of Medical Research, vol. 126, no. 3, pp. 216-219, 2007.

[36] V. D. Malini, "Characterization and antifungal susceptibility of Candida species: a preliminary study," Journal of Academic and Clinical Microbiology, vol. 2, no. 15, pp. 55-57, 2000.

[37] A. K. Ragini, B. Sandhya, D. Gayatri, and Indumati, "Characterization and antifungal susceptibility testing for Candida species in a tertiary care hospital," Journal of Health Sciences and Research, vol. 2, no. 2, pp. 1-7, 2011.
[38] D. Taura, M. Maje, A. Koki, and M. Musa, "Antifungal resistance among Candida species from patients with genitourinary tract infection at Muhammad Abdullahi Wase Specialist Hospital, Kano," Nigerian Journal of Basic and Applied Sciences, vol. 21, no. 1, pp. 33-38, 2013.

[39] G. Ginter, H. P. Soyer, and E. Rieger, "Vaginal yeast colonization and promiscuity. A study of 197 prostitutes," Mycoses, vol. 35, no. 7-8, pp. 177-180, 1992.

[40] A. K. Ako-Nai, O. O. Kassim, M. O. Adeniran, and O. Taiwo, "A study of urinary tract infections at Ile-Ife, Nigeria," East African Medical Journal, vol. 70, no. 1, pp. 10-14, 1993.

[41] J. M. Baeten, P. M. Nyange, B. A. Richardson et al., "Hormonal contraception and risk of sexually transmitted disease acquisition: results from a prospective study," American Journal of Obstetrics \& Gynecology, vol. 185, no. 2, pp. 380-385, 2001.

[42] I. B. Enweani, H. C. Gugnani, R. Okobia, and S. B. Ojo, "Effect of contraceptives on the prevalence of vaginal colonization with Candida species in Edo State, Nigeria," Revista Iberoamericana de Micologia, vol. 18, no. 4, pp. 171-173, 2001.

[43] M. A. Yusuff, A. Q. Chowdhury, A. N. I. Sattar, and M. Rahman, "Evaluation of the effect of contraceptives on prevalence of Candida species on vaginal candidiasis in Dhaka, Bangladesh," Bangladesh Journal of Medical Microbiology, vol. 1, no. 2, pp. 6164, 2007.

[44] S. S. Taiwo, A. B. Ashubiaro, A. I. Olugbenga-Bello, D. P. Oparinde, E. O. Asekun-Olarinmoye, and J. O. Ashiru, "Pattern of sexually transmitted diseases seen at Ladoke Akintola University Teaching Hospital, Osogbo, Southwestern Nigeria," Nigerian Journal of Genitourinary Medicine, vol. 3, no. 1-2, pp. 4-7, 2004.

[45] H. C. Gugnani, F. K. Nzelibe, P. C. Gini, W. O. Chukudebelu, and A. N. U. Njoku-Obi, "Incidence of yeasts in pregnant and nonpregnant women in Nigeria," Mycoses, vol. 32, no. 3, pp. 131-135, 1989.

[46] M. M. Barousse, B. J. Van Der Pol, D. Fortenberry, D. Orr, and P. L. Fidel Jr., "Vaginal yeast colonisation, prevalence of vaginitis, and associated local immunity in adolescents," Sexually Transmitted Infections, vol. 80, no. 1, pp. 48-53, 2004. 


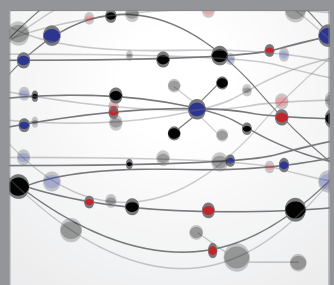

The Scientific World Journal
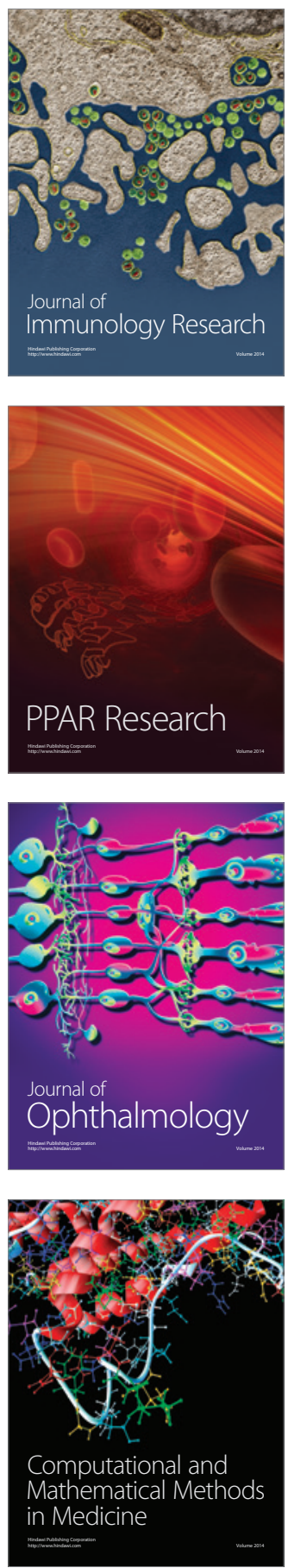

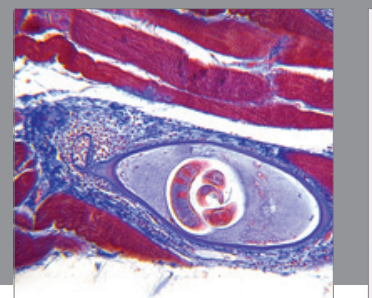

Gastroenterology

Research and Practice
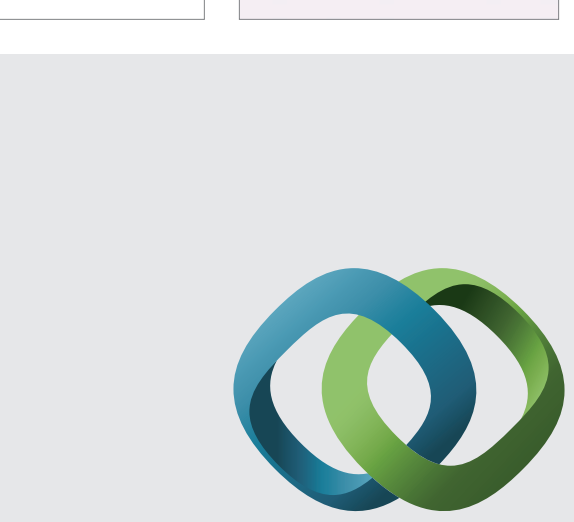

\section{Hindawi}

Submit your manuscripts at

http://www.hindawi.com
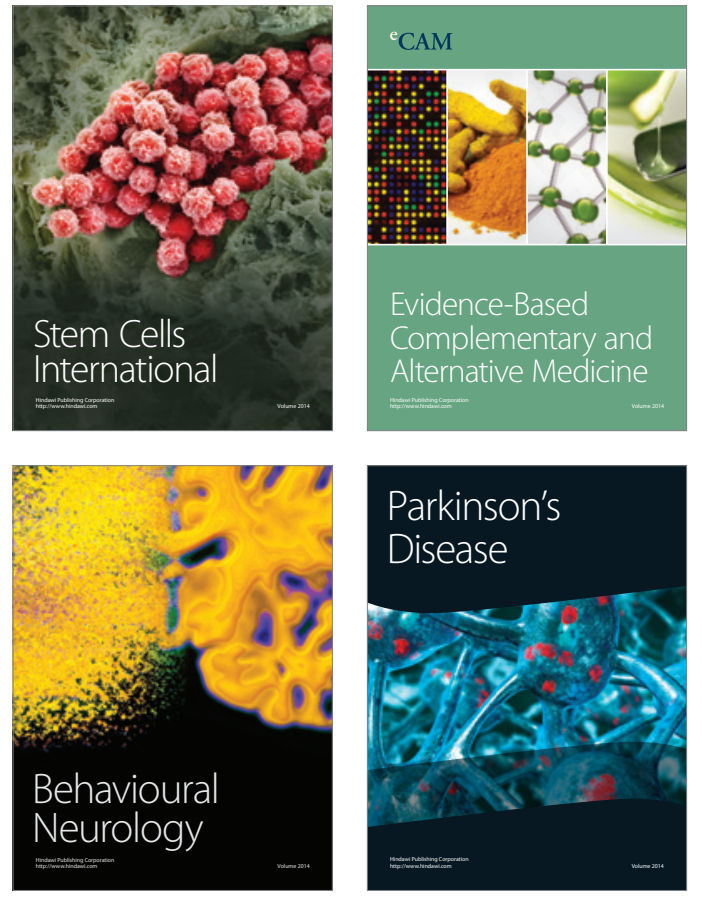
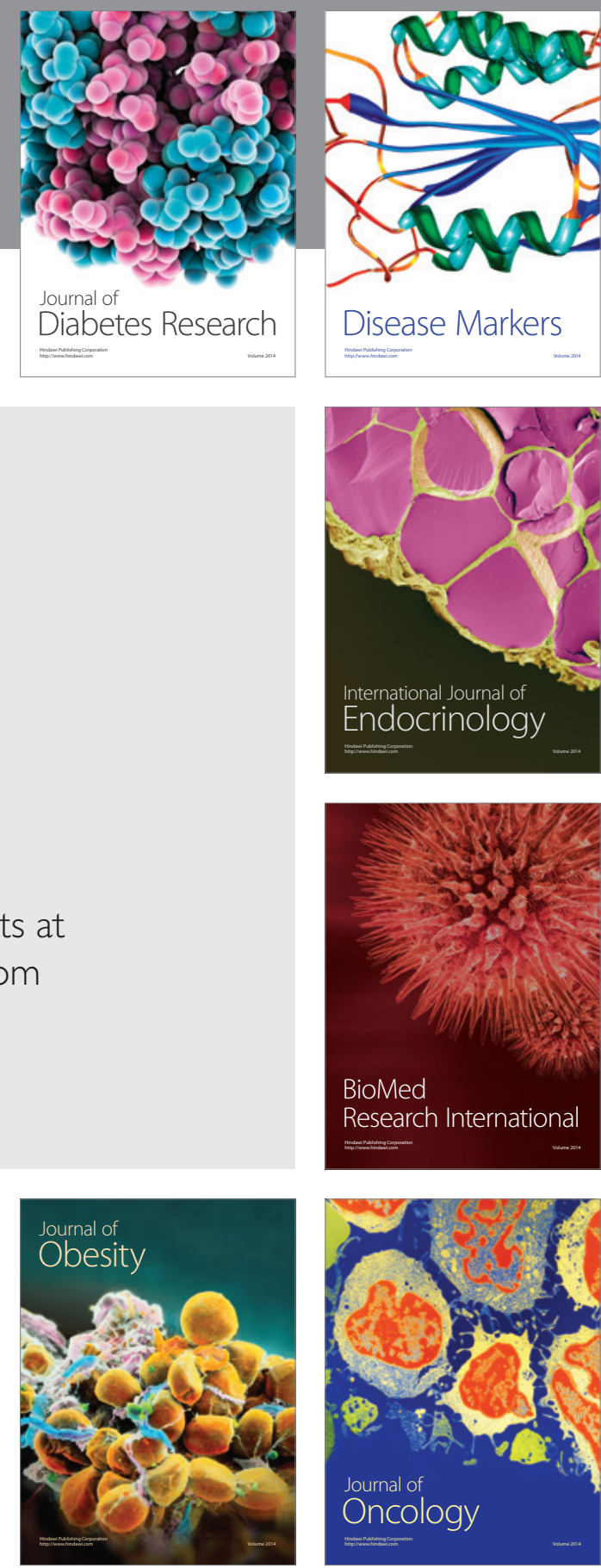

Disease Markers
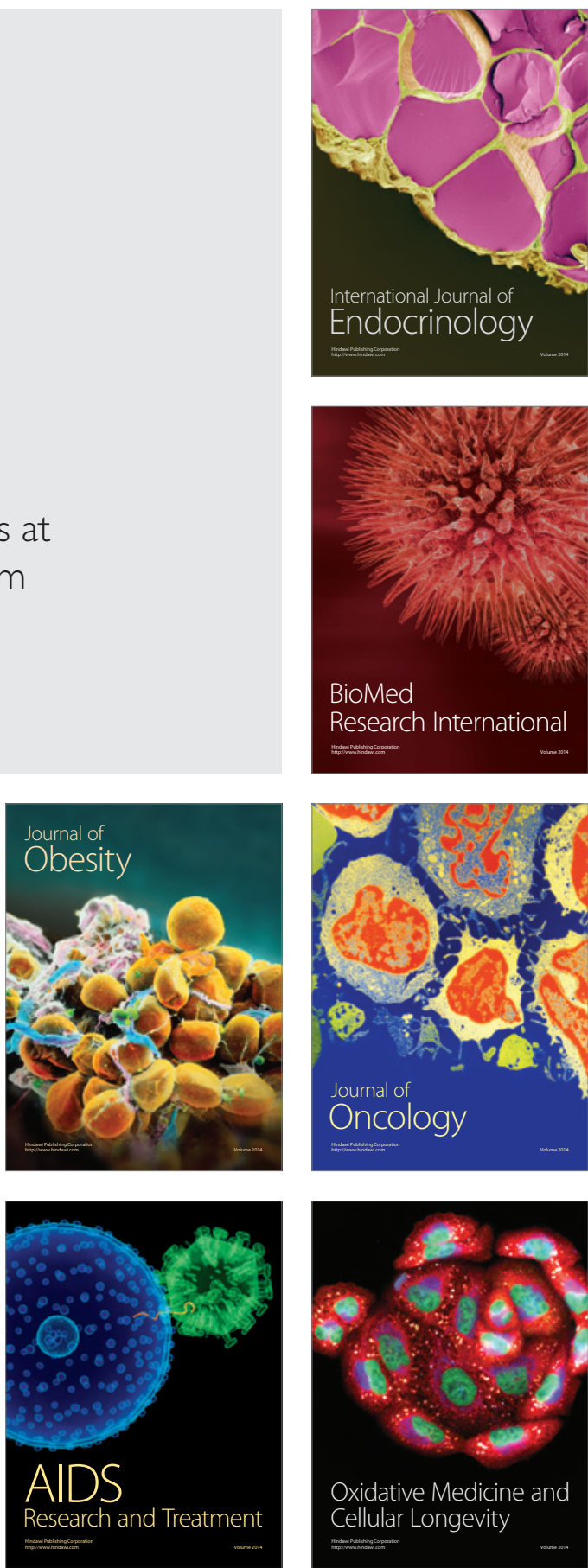